DOI: $10.12731 / 2306-1561-2013-4-3$

\title{
ANALYSIS OF PROCESS SIMULATION AND PROGRAMMING OF INDUSTRIAL ROBOTS
}

\section{Vasuhova S.A.}

\section{Abstract}

The article examines the processes of modeling and programming of industrial robots. As the object of investigation, the robot sorter, built on the basis of programmable controller LEGO NXT.

Keywords: bots, programmable controller, artificial intelligence, fuzzy logic, modeling.

\section{УДК 681.587}

\section{АНАЛИЗ ПРОЦЕССОВ МОДЕЛИРОВАНИЯ И ПРОГРАММИРОВАНИЯ ПРОМЫШЛЕННЫХ РОБОТОВ}

\section{Васюгова C.A.}

Аннотация

B статье анализируется прочессы моделирования $u$ программирования промышленных роботов. $B$ качестве объекта исследования выбран роботсортировщик, построенный на базе программируемого контроллера LEGO NXT.

Ключевые слова: роботы, манипуляторы, программируемый контроллер, искусственный интеллект, нечеткая логика, моделирование.

\section{Введение}

C каждым годом происходит успешное развитие автоматизации производственных процессов. Растет процент автоматизированных комплексов на заводах и предприятиях. Тема создания и моделирования роботов сегодня является наиболее популярной и актуальной. Человек пытается максимально ограничить свою деятельность, заменив ее с помощью компьютеризированной техники. За счет этого появляется большое количество методов проектирования и анализа разрабатываемых систем. Однако, наспех создаваемые системы не всегда могут порадовать своей работоспособностью из-за быстрых и частых поломок.

Промышленный робот представляет собой техническое устройство или комплекс технических устройств автоматического действия, оснащенные системой цифрового программного управления [2, 6 - 11]. Несмотря на всю автоматичность своих процессов, действия таких комплексов и устройств контролируется человеком. Многие 
из процессов требуют участия специалистов. В промышленных роботах совмещаются большая гибкость исполнительных органов, обладающих высокой подвижностью, и легкостью переналадки их двигательных функций. Роботы, предназначены для выполнения разнообразных работ при минимальном участии человека в акте управления. Они являются универсальными автоматами, в состав которых входят три основных функциональных узла: рабочие органы — «рука» или подвижная платформа, вычислительная машина (контроллер), управляющая ими, и устройства сбора информации о среде, сообщающие роботу об изменениях условий окружающей среды для способности адаптации к ней. Однако в промышленных условиях вероятность резких изменений условий окружающей среды невелика. Современные роботы обладают большим количеством устройств для сбора и обработки информации: инфракрасные датчики цвета, датчики передвижения, датчики прикосновения и т.д. Промышленный робот обладает встроенной памятью, а некоторые роботы имеют специальную систему обучения, принцип действия которой базируется на нейронных сетях.

Все эти факторы свидетельствуют о попытках создать совершенную машину с искусственным интеллектом, замещающую деятельность человека. Иными словами максимальная обособленность, достижение минимального вмешательства со стороны человека. Система, наделенная искусственным интеллектом, должна анализировать окружающую среду, создавать ее виртуальную модель и прогнозировать возможные способы решения поступающих задач в режиме "реального времени" [1, 3 - 5].

\section{Принцип работы промышленного робота}

Промышленный робот состоит из подвижной платформы, вычислительной машины и оснащен приспособлениями для сбора информации [2, 6 - 11]. Работа данного комплекса начинается с запуска управляющего устройства. После этого вычислительная машина (контроллер) обрабатывает и подает сигнал на вход двигателя, что приводит в действие робота, который с помощью устройств сбора информации выполняет необходимые действия. Наиболее наглядно эта система продемонстрирована на рисунке 1 , который содержит функциональную схему роботаманипулятора.

На рисунке продемонстрировано, как система управления, в соответствии с заданной программой, формирует и выдает на исполнительные устройства приводов (двигатели) управляющие воздействия ui. При необходимости она корректирует эти воздействия по сигналам Dxi, которые поступают в нее с датчиков обратной связи $[6,8$ - 11].

В составе робота есть механическая часть и система управления этой механической частью, которая в свою очередь получает сигналы от сенсорной части.

Современные роботы функционируют на основе принципов обратной связи, подчинённого управления и иерархичности системы управления роботом. 


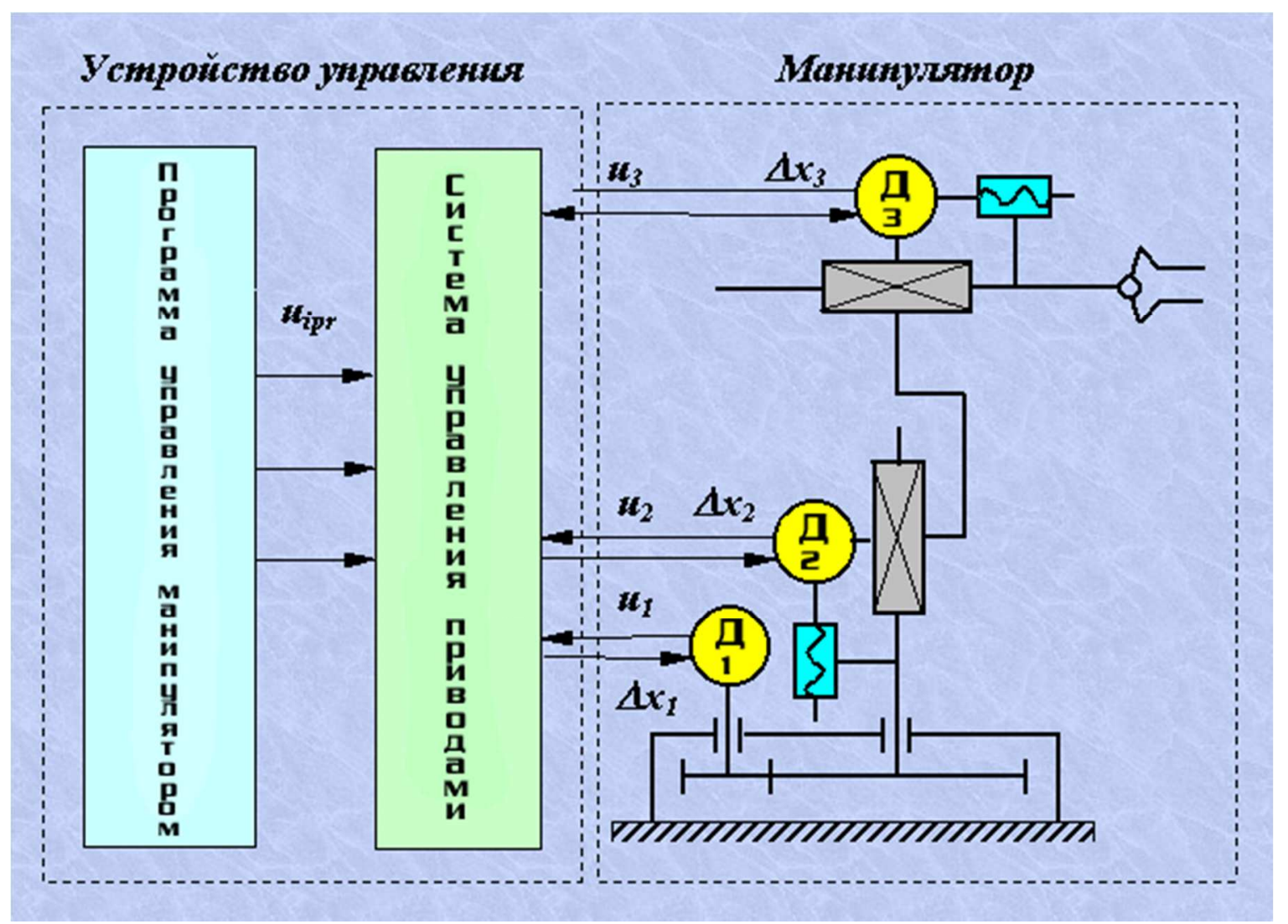

Рисунок - 1. Функциональная схема промышленного робота

Иерархия системы управления роботом подразумевает деление системы управления на горизонтальные слои, управляющие общим поведением робота, расчётом необходимой траектории движения манипулятора, поведением отдельных его приводов, и слои, непосредственно осуществляющие управление двигателями приводов [2, 6 - 11].

\section{Процесс моделирования промышленного робота}

Немаловажным этапом в проектировании и создании каких- либо устройств является создание алгоритмов работы будущих устройств. Данный этап поможет последовательно определить процесс проектирования и программирования, а также, составить алгоритм работы создаваемого устройства. Для анализа всех этих процессов была сконструирована рабочая модель промышленного робота- сортировщика (рисунок $2,3)$ на основе элементов конструктора LEGO и программируемого котроллера LEGO NXT.

Робот-сортировщик имеет два серво-мотора, схема которых представлена на рисунке 3, один датчик света, контроллер NXT. Для соединения всех элементов к контроллеру используются кабели разной длины. 


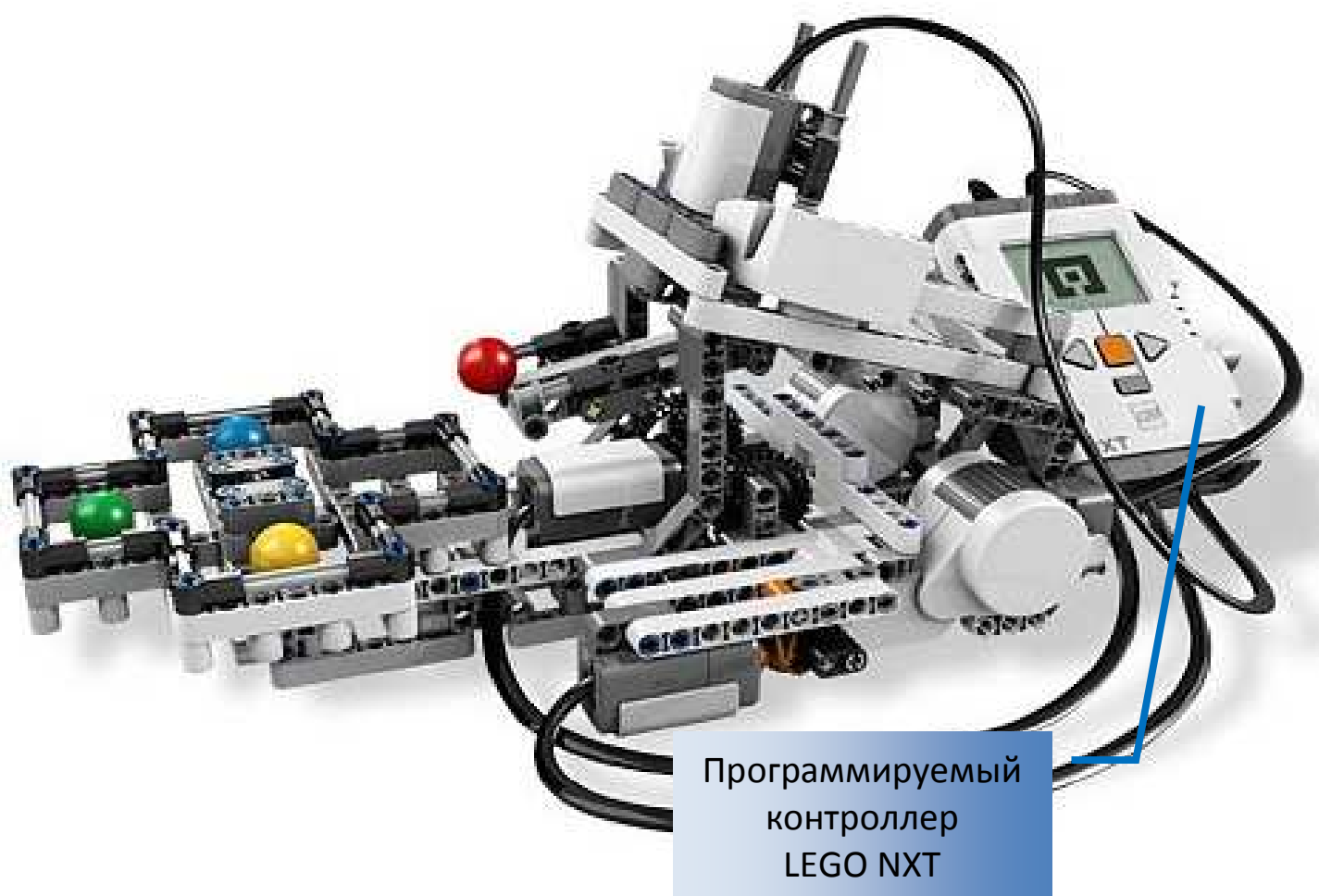

Рисунок 2- Модель промышленного робота-сортировщика LEGO

Тахометр для встроенного датчика оборотов

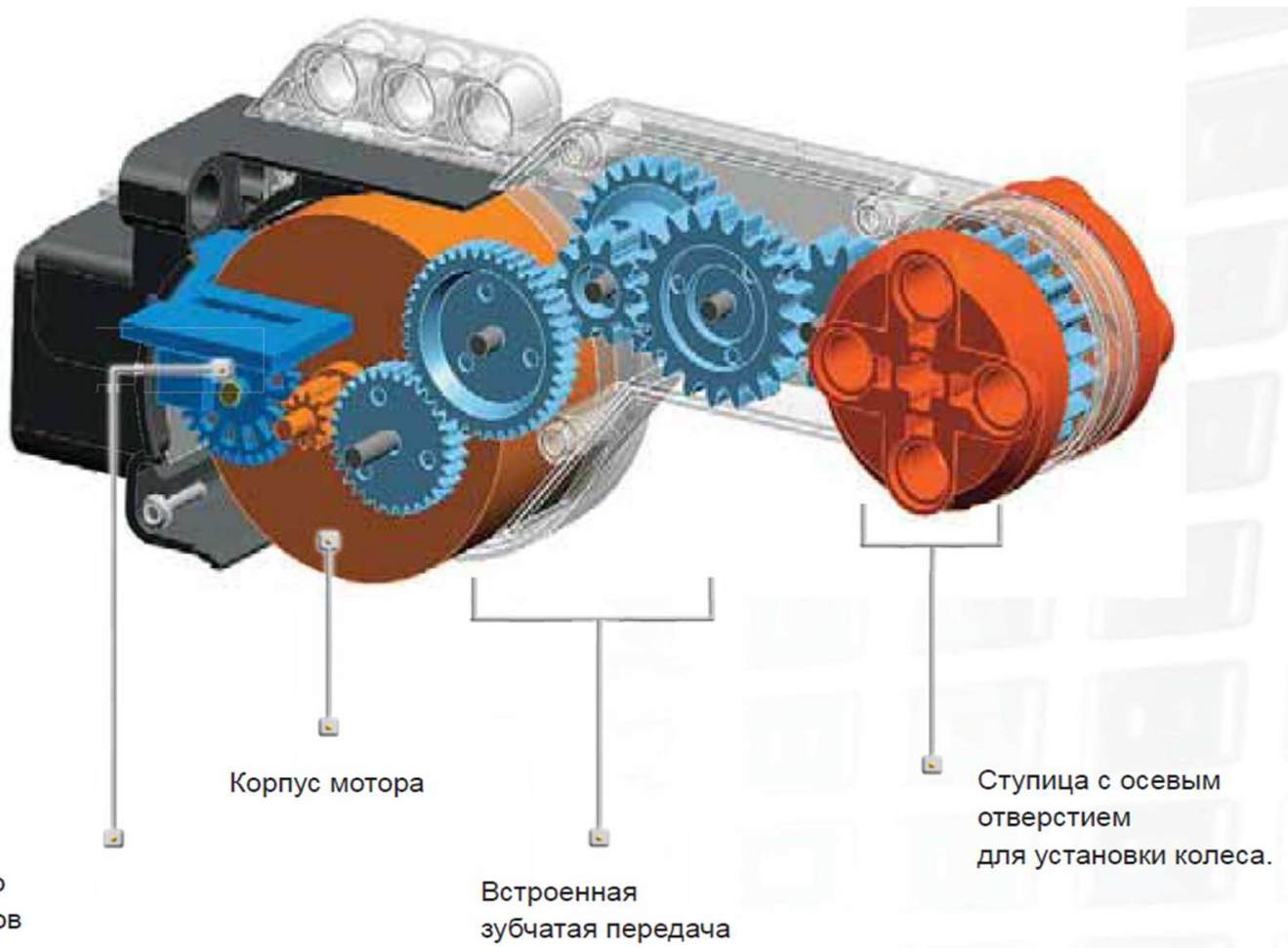

Рисунок 3 - Сервомотор

Для сортировки создана платформа, где будут располагаться цветные предметы. Платформа поднимается, и предметы по очереди попадают в проем, который просвечивается световым датчиком. После определения цвета открывается лопасть, располагающаяся в нижней части платформы и предмет скатывается на подвижную 
платформу с ячейками. Движение платформ с не отсортированными предметами и с ячейками обеспечивается двумя моторами.

Каждый мотор оснащён встроенным датчиком оборотов, что позволяет очень точно управлять движениями робота. Датчик производит измерения в градусах или в количестве полных оборотов (точность измерения +/- один градус). Один полный оборот соответствует 360 градусам, следовательно, если вы зададите мотору поворот на 180 градусов, его вал сделает пол оборота.

Главным элементом системы контроля и управления являются контроллеры. Контроллер LEGO NXT (см. рисунок 2), позволяет запрограммировать разработанный нами робот. Контроллер - это управляющее устройство, которое способно на аппаратном уровне обрабатывать алгоритмы, заложенные пользователем.

Контроллер может работать как в сетевом, так и в автономном режиме. Сетевой режим подразумевает работу с подключением контроллера к персональному компьютеру, и следовательно управление с помощью программной консоли. В случае потери связи с компьютером или отключении сети питания, контроллер автоматически переходит в автономный режим работы, продолжая функционировать и отрабатывать алгоритмы в полном объеме, используя при этом свой встроенный блок питания. При работе в автономном режиме контроллер сохраняет все данные в объеме, который зависит от размера встроенной памяти, и после восстановления связи с компьютером, передает всю информацию в основную базу данных. В автономном режиме контроллер может работать около четырех часов.

Приступая к программированию робота необходимо определить основные задачи, которые устройство будет решать в дальнейшем.

\section{Разработка алгоритмов управления роботом}

Немаловажным этапом в проектировании и создании каких- либо устройств является создание алгоритмов работы будущих устройств. Данный этап поможет последовательно определить процесс проектирования и программирования, а также, составить алгоритм работы создаваемого устройства. Метод предварительного программного анализа формулирует работу системы по следующему характеру (рисунок 4).

На систему управления (контроллер) подается условие или значение(задача), после чего оно попадает в анализатор и приравнивается к const. Далее происходит ввод требуемых данных и они посылаются в решатель, который осуществляет выполнение необходимых математических, логических и программных действий. Решатель посылает полученные данные (Rez) в анализатор, который сравнивает принятые данные с const: если данные не равны (Rez=const), то они пересылаются в регуляторблок, содержащий алгоритмы наращивания или уменьшения при различных условиях и типах данных. 


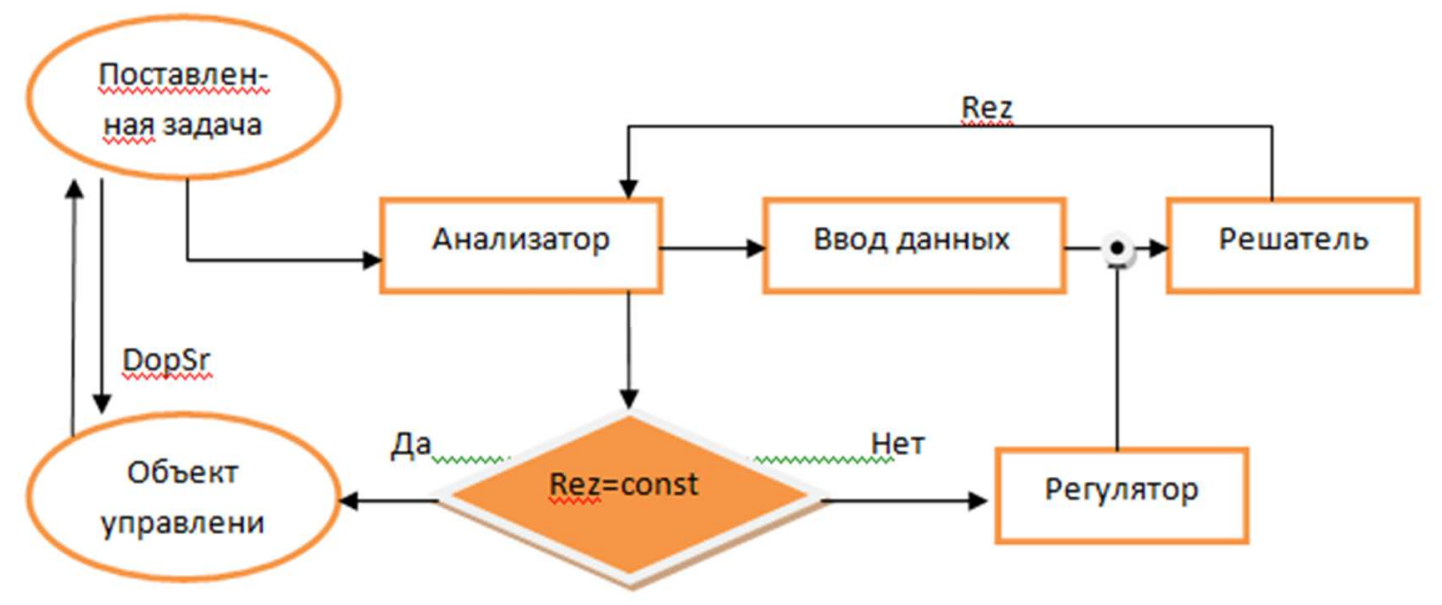

\section{Рисунок 4 - Схема процесса работы управляющего устройства}

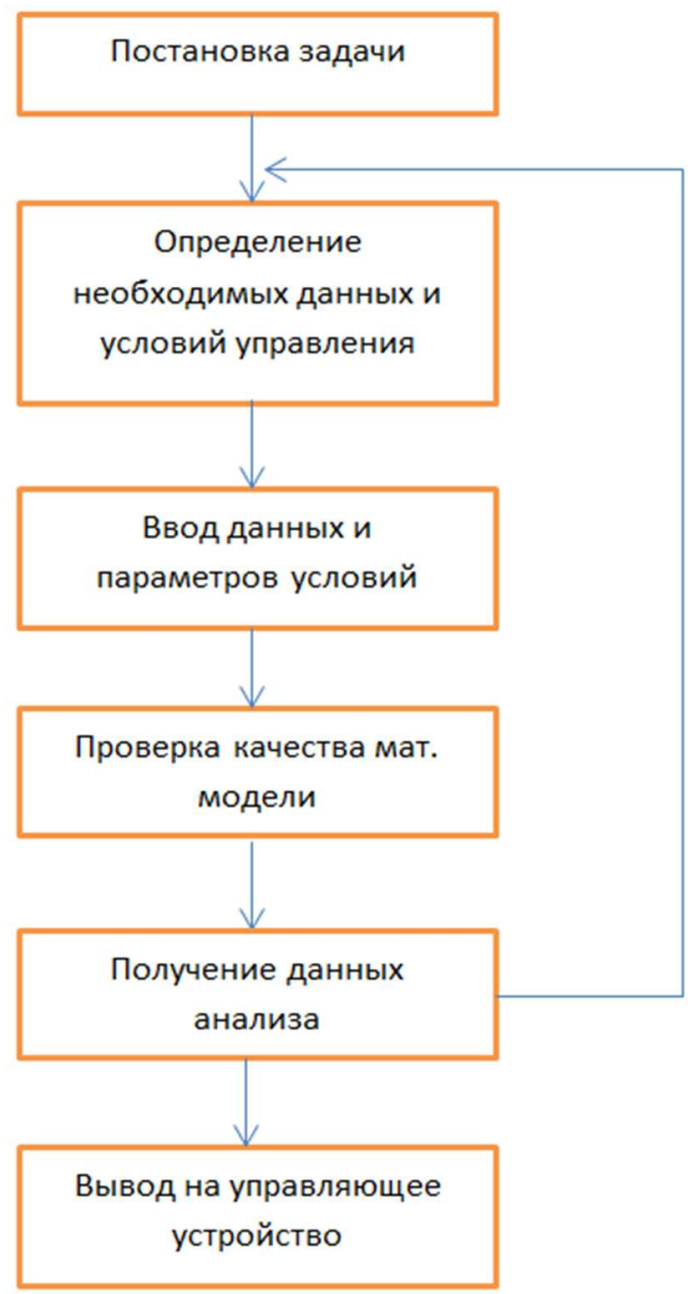

Рисунок 5 - Алгоритм процесса проектирования и программирования робота

Регулятор сравнивает полученный результат с желаемым, после чего выполняет действие приближения к желаемому результату. Если данные в анализаторе равны, то они подаются на объект управления, который в свое время подает отчет о проделано 
работе и конечном результате на выходе, который дополнительно сравнивается (DopSr) с поставленной задачей.

Данную систему можно назвать интеллектуальной, т.к. она позволяет просчитывать несколько комбинаций решения и самостоятельно искать способы решения проблем.

Алгоритм создания и программирования промышленного робота по методу предварительного программного анализа предлагается такой (рисунок 5).

После постановки задачи необходимо определить данные и условия, которые будут использоваться в реализации программного процесса. После определения данных осуществляется ввод имеющихся данных в среду разработки и анализа качества будущей системы. После чего необходимо протестировать качество создаваемого алгоритма управления системы. Все результаты, полученные по итогам тестирования качества, следует проанализировать, и если система не надежна, выполнить пересмотр определенных данных и условий, выполнить их коррекцию и вновь проверить на качество и безотказность. Если алгоритм управления системы качественный, подать его на управляющее устройство.

Блок-схема такого алгоритма будет следующей (рисунок 6).

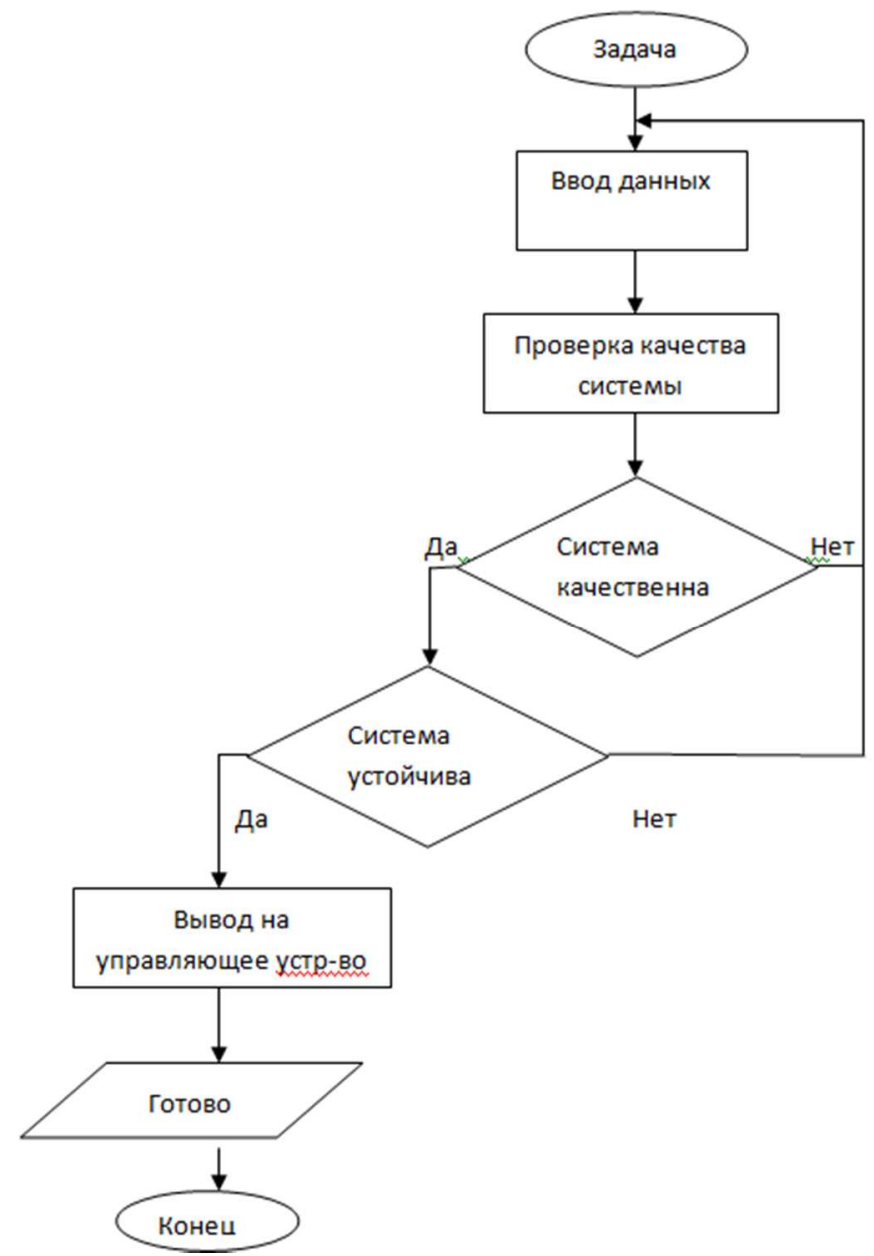

Рисунок 6 - Блок-схема алгоритма процесса проектирования и программирования робота 
Данный метод показал, что процесс разработки логических систем является более объемным по охвату разрабатываемой области, и менее емким по отношению к количеству процессов, которые необходимо сделать на различных этапах разработки системы. Это говорит о том, что следуя этому алгоритму, мы сможем разработать более качественную и более устойчивую систему.

После эксплуатации нашего робота в различных условиях, возникли проблемы со световыми помехами. Сегодня это наиболее распространенная проблема в эксплуатации технических устройств.

\section{Решение проблемы подавления световых помех}

При выполнении заложенных в себя функций, робот сталкивается с внешней средой, которая может внести изменения в алгоритм работы робота. Внешние факторы можно классифицировать на факторы, которые влияют на результат работы алгоритма и факторы, не влияющие на результат работы алгоритма.

Внешние факторы, влияющие на результат работы алгоритма можно разделить на 2 типа - факторы, которые являются факторами той же природы, что и информация, получаемая из внешней среды и факторы, иной природы. Примером внешнего фактора той же природы, что и получаемые данные является струя воздуха из системы охлаждения робота, попадающая на датчик температуры. Солнечный свет, нагревающий датчик является внешним фактором другой природы. Искусственное освещение, которое тоже нарушает работу робота. Существует ряд способов по решению таких проблем. На рисунке 7 представлена схема решения проблем влияния внешних факторов:

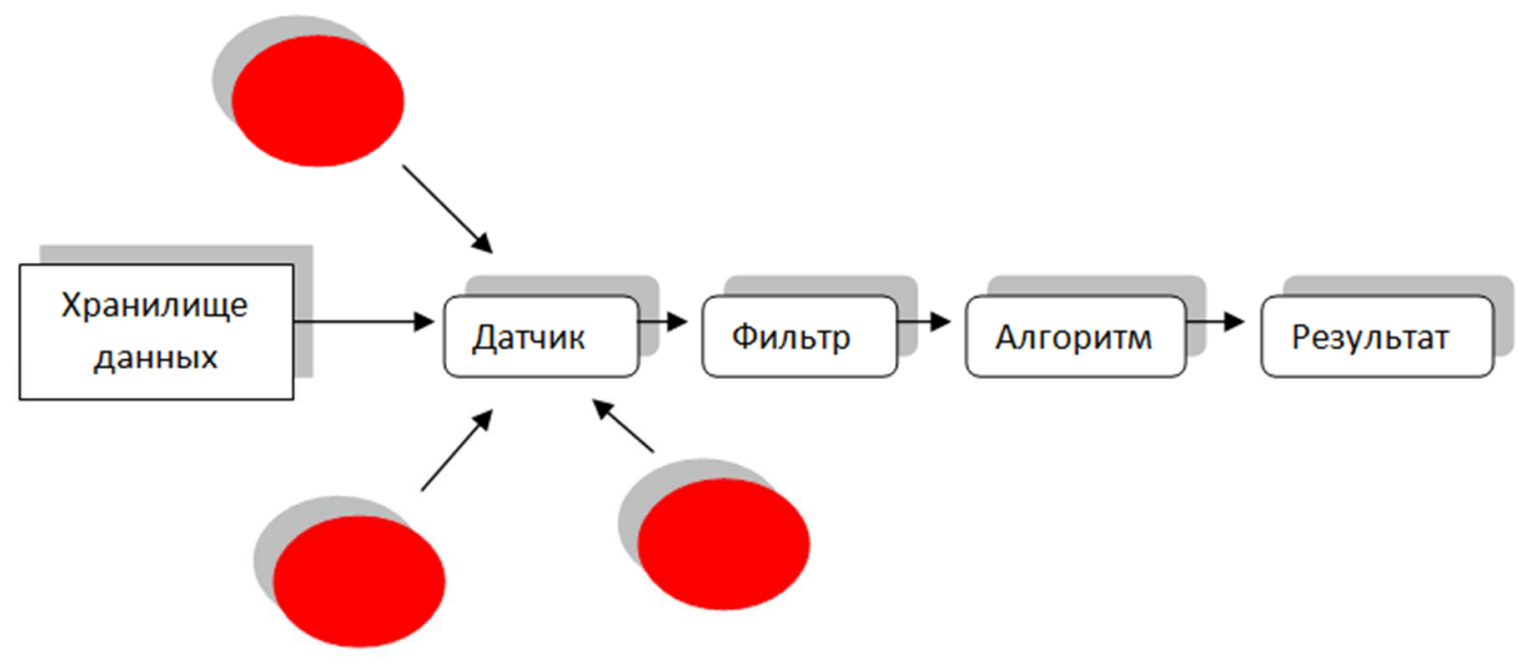

\section{Рисунок 7 - Схема решения проблем влияния внешних факторов}

Круги на рисунке отвечают за расположение источников внешних воздействий. После блока «Датчик» следует блок «Фильтр», что позволит минимизировать процент воздействия внешней среды на конечный результат. При моделировании робота, 
фильтр может представлять различные варианты как технических, так и конструкторских решений.

Вышеизложенные теоретические выкладки применены к прототипу промышленного робота-сортировщика, построенного на базе контроллера LEGO NXT. Роботу подавался шарик одного из 4-х цветов - желтого, красного, зелёного и синего. Данный робот с помощью датчика определял цвет подаваемого ему шарика и помещал шарик в предназначенный ему отсек. В затемнённом помещении ошибок в работе робота не наблюдалось, однако при работе робота в условиях изменяемой освещённости стали наблюдаться сбои в работе робота. В частности, он периодически путал желтый, красный и зелёный цвета, при этом ошибок с определением синего цвета не наблюдалось. Очевидно, что внешним фактором, влияющим на результат работы алгоритма, был искусственный источник света. Эта помеха той же природы, что и входные данные. Исходя из этого, существовало 3 пути устранения данной помехи:

- изменить чувствительность датчика;

- изменить или устранить источники искусственного освещения;

- создать помехи на пути искусственного света (фильтры).

Путём изменения алгоритма проблема не решается, изменение чувствительности датчика, по сути, приводит к замене самого датчика, что тоже невозможно ввиду отсутствия другого датчика. Соответственно, остаётся только путь создания помех на пути искусственного источника света. Для этих целей был сконструирован компактный короб, который помещается сверху датчика и закрывает поступление света как сверху, так и с боков. После тестирования нового фильтра, процент выдаваемых ошибок снизился втрое, что свидетельствует о целесообразности использования подобных фильтров на датчиках устройств.

\section{Заключение}

В ходе исследования был проведен анализ ряда проблем, связанных с разработкой роботизированных комплексов и устройств на примере созданного роботасортировщика. Был предложен метод предварительного программного анализа. Данный метод продемонстрировал, что процесс разработки логических систем является более объемным по охвату разрабатываемой области, и менее емким по отношению к количеству процессов, которые необходимо сделать на различных этапах разработки системы. Была рассмотрена наиболее распространенная проблема в эксплуатации роботов- световые помехи.

\section{Список информационных источников}

[1] Васюгова С.А. Исследование перспектив и проблем интеграции человека с компьютером: искусственный интеллект, робототехника, технологическая сингулярность и виртуальная реальность / А.В. Остроух, С.А. Васюгова, М.Н. Краснянский, А. Самаратунга // Перспективы науки. - 2011. - № 4(19). - С. 109 112. 
[2] Васюгова С.А. Моделирование поведения интеллектуального робота / С.А. Васюгова, Чаудхари Раджа Рам, О.И Максимычев, А.М. Васьковский // В мире научных открытий. - 2012. - №2.6 (26). - С. 110 - 114.

[3] Варламов О.О., Васюгова С.А., Остроух А.В. Применение систем виртуальной реальности для дистанционного обучения и создания миварных электронных образовательных ресурсов // ЭЛЕКТРОННОЕ ОБУЧЕНИЕ И ДИСТАНЦИОННЫЕ ОБРАЗОВАТЕЛЬНЫЕ ТЕХНОЛОГИИ. - 2013. - № 1; URL: eodot.esrae.ru/1-9 (дата обращения: 27.09.2013).

[4] Белоусова А.И. Подход к формированию многоуровневой модели мультиагентной системы с использованием миваров / А.В. Остроух, А.И. Белоусова, М.Н. Краснянский, О.О. Варламов // Перспективы науки. - 2011. - № 5(20). - С. 57 - 61.

[5] Варламов О.О. Анализ перспектив использования технологий виртуальной реальности в дистанционном обучении / А.В. Остроух, Я.Г. Подкосова, О.О. Варламов, М.Н. Краснянский // Вопросы современной науки и практики. Университет им. В.И. Вернадского. - 2011. - № 2(33). - С. 104 - 111.

[6] Остроух, А.В. Информационные технологии в научной и производственной деятельности / [ред. А.В. Остроух] - М: ООО "Техполиграфцентр", 2011. - 240 с. ISBN 978-5-94385-056-1.

[7] Остроух, А.В. Основы построения систем искусственного интеллекта для промышленных и строительных предприятий / А.В. Остроух - М.: ООО «Техполиграфцентр», 2008. - 280 с. - ISBN 978-5-94385-033-2.

[8] http://ru.wikipedia.org/wiki/Промышленный робот

[9] http://www.autowelding.ru/index/promyshlennye_roboty/0-67

[10] http://haselab.info/soinn-e.html

[11] http://training-site.narod.ru/industrial.html 